\title{
Sintomas psiquiátricos e comportamentais da demência
}

Frederico Simões do Couto, ${ }^{*}$ Alexandre de Mendonça*

\section{RESUMO}

Para além das alterações cognitivas, as demências incluem outras manifestações sintomáticas de enorme importância. Estas manifestações de cariz psiquiátrico e comportamental têm sido designadas, no seu conjunto, por sintomas psiquiátricos (ou psicológicos) e comportamentais da demência (SPCD). OS SPDC são bastante importantes por várias razões e são uma razão importante de consulta dos Clínicos Gerais/Médicos de Família.

Alguns destes sintomas tendem a ocorrer simultaneamente, em factores, como a psicose (inclui a agitação e a agressividade), o humor (depressão e ansiedade), o comportamento frontal (desinibição e euforia), a apatia e a insónia.

A causa destes sintomas pode ser a própria demência e, nalgumas situações, podem constituir elementos nucleares do seu diagnóstico. Todavia, a possibilidade de serem causados por uma outra doença médica ou por fármacos deve ser activamente investigada, especialmente se se observarem algumas características sintomatológicas particulares. A exclusão de uma causa secundária (não demencial) é obrigatória.

Em termos de tratamento, e excluídas outras causas secundárias, deve ser instituída uma terapêutica antidemencial específica, já que há evidência que os inibidores da acetilcolinesterase e a memantina melhoram, não só os sintomas cognitivos, mas também os SPCD nalgumas demências. Em segundo lugar, devem ser instituídas medidas não farmacológicas. Todavia, se houver urgência no tratamento ou se os sintomas foram muito intensos, pode ser necessário iniciar de imediato tratamento farmacológico sintomático específico.

Deve ser identificado o sintoma ou os sintomas problemáticos e ser efectuada terapêutica farmacológica dirigida. Apesar de os ensaios clínicos realizados com o objectivo de avaliar a eficácia das terapêuticas para os SPCD serem escassos e por vezes de qualidade insuficiente, é de extrema importância tratar estes sintomas. É necessário também instruir os cuidadores, pois são pessoas que habitualmente não possuem competência técnica para lidar com estes fenómenos.

Qualquer SPCD de difícil controlo, especialmente se não melhorar após as medidas terapêuticas aqui descritas, poderá ser referenciado a uma consulta de Psiquiatria ou Neurologia, com a indicação de «SPCD [especificar] de difícil controlo após tratamento com [especificar]».

Palavras-chave: Sintomas Psiquiátricos e Comportamentais da Demência; Diagnóstico; Tratamento, Antipsicóticos; Psicotrópicos.

\section{INTRODUÇÃO}

$\mathrm{P}$ ara além das alterações cognitivas (i.e. para além das funções necessárias directamente ao processo do conhecimento, como a atenção, a concentração, a memória, a linguagem, etc.), as demências incluem outras manifestações sintomáticas de enorme importância. Algumas destas manifestações de cariz psiquiátrico e comportamental têm sido designadas, no seu conjunto, por sintomas psiquiátricos (ou psicológicos) e comportamentais da demência (SPCD). ${ }^{1}$

Estes SPCD incluem apatia, agressividade/agitação,

*Grupo de Demências, Serviço de Neurologia e Laboratório de Neurociências, Faculdade de Medicina e Instituto de Medicina Molecular, Lisboa. ansiedade, depressão, sintomas psicóticos (delírios e alucinações), desinibição, euforia, actividades sem objectivo (remexer em objectos, vaguear), alterações do sono e outras alterações da percepção., ${ }^{2,3}$

Estes sintomas têm importância porque (1) requerem um diagnóstico diferencial com outras doenças orgânicas, (2) existem terapêuticas específicas com comparticipação generalizada para muitos deles, (3) e porque constituem, com frequência, um factor de desgaste para os cuidadores. ${ }^{4,5}$ De facto, os SPCD têm um impacte muito importante nos doentes, nomeadamente institucionalização mais precoce, aumento do uso de fármacos, pior evolução dos sintomas cognitivos, e também nos cuidadores, com maior frequência de de- 
pressão e de outras doenças psiquiátricas e risco de serem agredidos fisicamente. ${ }^{6}$ Alguns destes sintomas, em particular os sintomas psicóticos, têm sido associados a uma evolução mais rápida.

Daqui resulta que os Clínicos Gerais/Médicos de Família terão frequentemente de lidar com estes sintomas e com as suas consequências.

\section{DIAGNÓSTICO}

Os SPCD devem ser avaliados clinicamente, com perguntas directas ao doente e ao cuidador, já que, muitas vezes, são subvalorizados até ao surgimento de uma crise. Deve ser caracterizado o tipo de sintoma (pode auxiliar na organização do plano para o doente a sua classificação nos factores abaixo referidos), a sua intensidade, a existência de flutuação, a repercussão no doente e cuidadores e outros sintomas acompanhantes.

Existem algumas escalas (para efeitos maioritariamente de investigação, embora existam recomendações para serem usadas na prática clínica) para objectivação e quantificação da sintomatologia, como p. ex. o NeuroPsychiatric Inventory (NPI), ${ }^{7}$ que está traduzido para português. ${ }^{8}$

Alguns destes sintomas tendem a ocorrer simultaneamente, em factores, e apresentam alguns aspectos comuns em termos das características clínicas, gravidade e resposta ao tratamento. Os factores habitualmente descritos são psicose (inclui a agitação e a agressividade), humor (depressão e ansiedade) e comportamento frontal (desinibição e euforia). ${ }^{9}$ Outros sintomas importantes são a apatia e a insónia.

\section{Factor Psicose}

Inclui delírios, alucinações, agitação e irritabilidade. Os delírios, habitualmente fragmentados e pouco sistematizados, são de roubo, sósia (pessoas familiares foram substituídas por outras), presença de estranhos ou, mais raramente, de perseguição. Muitas vezes têm origem na perda de memória, já que os doentes não se recordam onde põem os objectos (e acreditam terem sido roubados) ou não reconhecem as caras dos familiares.

As alucinações podem ser visuais na demência com corpos de Lewy (DCLewy) - que nesta doença são vívidas e complexas, ou auditivas mais simples (p. ex. ouvir o nome). Também pode ocorrer a chamado sensação de presença, que é difícil de relacionar com uma modalidade sensorial, e que se define como a sensação de que está alguém presente, mas que não é identificada. Esta é, aliás, uma experiência que pode ocorrer em pessoas saudáveis.

A agitação e a agressividade são os sintomas com maior impacte nos cuidadores e estão associados a frequentes pedidos de institucionalização.

\section{Factor humor}

Este factor inclui a depressão e a ansiedade.

A depressão é muito comum na demência, podendo atingir valores entre $20-50 \%$ na doença de Alzheimer (DA). ${ }^{3}$ É mais frequente na fase inicial da DA e pode, inclusive, ser uma manifestação inicial desta doença.

A depressão e a demência apresentam alguns sintomas comuns, pelo que o diagnóstico de depressão numa pessoa com demência (como aliás no idoso em geral) apresenta algumas especificidades. Deve ser diagnosticada com base na tristeza, sentimentos ou ideias de menos valor sobre o próprio, o mundo e o futuro, pessimismo, ideias de suicídio (devem ser directamente perguntadas - é um receio infundado que isso aumenta o risco de suicídio) e a diminuição do prazer (frases como já nada me interessa). As alterações da concentração, atenção e memória, a lentificação psicomotora, as alterações do sono e as alterações do apetite devem ter um papel limitado no diagnóstico, ${ }^{3}$ precisamente porque podem ser sintomas comuns às duas doenças. Pelo contrário, sintomas somáticos, nomeadamente dolorosos, são elementos de diagnóstico da depressão no idoso e no demente.

A ansiedade manifesta-se sobretudo por sintomas físicos de desconforto, tensão muscular, insónia inicial, palpitações e dispneia.

\section{Factor comportamento frontal}

Neste factor estão agrupados os comportamentos desinibitórios. Os doentes comportam-se de forma impulsiva e inapropriada, manifestando instabilidade, distractilidade e pouca capacidade crítica para as convenções sociais. Podem apresentar choro, euforia ou agressividade explosivas e inusitadas, comportamentos de desinibição sexual, comer compulsivamente (com perda de normas), agitação motora, familiaridade compulsiva, ou até consumos de álcool e violência em relação a outros. 


\section{Apatia}

A apatia traduz-se por falta de interesse, de iniciativa e de actividade em vários aspectos da vida, com diminuição da resposta emocional, da expressão facial ou das inflexões da voz. Deve ser distinguida da depressão, pois a apatia não se acompanha de tristeza, nem de conteúdos pessimistas ou negativos.

\section{Problemas de sono}

Observados em 25-43\% dos doentes com demência, ${ }^{3}$ estão habitualmente relacionadas com outros SPCD, embora possam ter características sugestivas do diagnóstico de DCLewy.

\section{DIAGNÓSTICO DIFERENCIAL}

A causa destes sintomas é, frequentemente, a própria demência, e nalgumas situações (como a Demência com Corpos de Lewy ou a Demência Fronto-Temporal) podem constituir elementos nucleares do diagnóstico. Todavia, a possibilidade de serem causados por uma outra doença médica ou por fármacos deve ser investigada, especialmente se estes sintomas surgirem, ou se se agravarem, de forma súbita e inesperada, se houver alteração da consciência (diminuição do estado de vigília, como p. ex. sonolência), ou se surgirem em concomitância outros sintomas (sinais focais, asterixis, desorganização do pensamento, alterações da marcha, ou alucinações visuais, por exemplo).

Assim, a exclusão de uma causa secundária (não demencial) é obrigatória. São causas frequentes as infecções (especialmente respiratórias e urinárias; precaução com a possibilidade de sépsis), alterações hidro-electrolíticas, descompensações endócrino-metabólicas (p. ex., da diabetes), doenças cardiovasculares (descompensação de insuficiência cardíaca; atenção ao tromboembolismo pulmonar e ao enfarte agudo do miocárdio), neoplasias (pulmonares e digestivas), úlceras gastrointestinais, escaras, fracturas, acidentes cérebro-vasculares, entre outras. ${ }^{1,10,11}$ Os SPCD também podem ser causados por fármacos, directamente ou por abstinência. Os fármacos potencialmente responsáveis são muitos, embora os mais frequentemente envolvidos sejam aqueles com actividade anticolinérgica (antiparkinsónicos, antidepressivos tricíclicos, alguns antipsicóticos, alguns medicamentos para a incontinência urinária, ou com actividade sobre o intestino), benzodiazepinas, alguns anti-hipertensores, alguns antibióticos, corticosteróides, estimulantes do SNC (teofilina, cafeína, etc), antiepilépticos e alguns anti-inflamatórios não esteróides. A abstinência é mais frequente a benzodiazepinas e a medicações analgésicas (tramadol, por exemplo).

\section{TRATAMENTO}

Em termos de tratamento, e excluídas outras causas secundárias, deve ser instituída uma terapêutica antidemencial específica, já que há evidência de que os inibidores da acetilcolinesterase e a memantina melhoram, não só os sintomas cognitivos, mas também os SPCD na Doença de Alzheimer, e a rivastigmina na Demência com Corpos de Lewy. ${ }^{6}$ Os inibidores da acetilcolinesterase não devem ser usados na Demência Fronto-Temporal (DFT), pois podem agravar os SPCD, apesar de existirem alguns estudos que indicam o contrário. Quanto às outras demências, e apesar de serem usados correntemente, a evidência para o seu uso baseia-se essencialmente em casereports ou pequenas séries.

Devem ser instituídas medidas não farmacológicas. ${ }^{1,4}$ De facto, muitos destes sintomas são desencadeados por alterações no ambiente (p.ex. mudança de cuidador ao fim de semana, volume da televisão muito alto, excesso de estimulação), pelo que a identificação e a correcção destas alterações pode levar à melhoria dos SPCD.

Todavia, se houver urgência no tratamento ou se os sintomas forem muito intensos, pode ser necessário iniciar de imediato tratamento farmacológico sintomático específico.

Deve ser, quando possível, identificado o sintoma ou os sintomas problemáticos e ser efectuada terapêutica farmacológica dirigida. Apesar de os ensaios clínicos realizados com o objectivo de avaliar a eficácia das terapêuticas para os SPCD serem escassos e por vezes de qualidade insuficiente, é de extrema importância não deixar de os tratar, pois, como já foi referido atrás, estão associados a um grande sofrimento. É necessário também instruir os cuidadores, pois são pessoas que habitualmente não possuem competência técnica para lidar com estes fenómenos e só o bom senso não é suficiente.

\section{Factor Psicose}

Para além das causas referidas, a agitação e a agressivi- 
dade estão relacionadas com desocupação, desconforto, isolamento, dor, depressão ou necessidade de actividade. $\mathrm{O}$ tratamento não farmacológico para a agitação e psicose deve ser individualizado e identificar algumas destas causas. Podem ser iniciadas medidas simples como a estimulação e ocupação adequadas, aumento do espaço para se deslocar e do conforto, ou actividades de socialização controlada (p.ex. ver vídeos ou fotografias da família, envolvimento em actividades parecidas com a profissão anterior, fisioterapia, ou massagens). Em termos da atitude a adoptar, esta não deve ser confrontativa ou de forma a pôr em evidência os défices, sendo preferíveis a empatia, o toque gentil e uma postura de não recriminação.

Para o tratamento farmacológico da agitação e da agressividade, podem ser usadas benzodiazepinas, antipsicóticos (primeira e segunda gerações), trazodona, ou anticonvulsivantes.

As benzodiazepinas devem ser prescritas em doses mais baixas do que as usadas em pessoas mais jovens, e devem ser preferidas as de semi-vida curta. Fármacos de semi-vida longa, como o diazepam, têm sido associados a fenómenos de impregnação (em que a velocidade de administração é superior à da eliminação), com aumento do risco de quedas, fracturas e mortalidade geral. Assim, é em geral sempre preferível o lorazepam, desde que se tenham em conta os efeitos moderadamente sedativos desta benzodiazepina.

A trazodona é um antidepressivo de estrutura tricíclica, mas sem efeitos anticolinérgicos e com uma actividade sedativa moderada. Há vários estudos, embora nem sempre consistentes, evidenciando que a administração deste fármaco, na dose de 50 a 100 mg à noite, reduz a agitação e agressividade diurnas. Este fármaco tem particular interesse na DFT.

Não devem ser escolhidos antipsicóticos com efeitos anticolinérgicos (como a cloropromazina, a ciamemazina ou a levomepromazina), pois agravam os sintomas cognitivos. Tem havido uma enorme controvérsia sobre o risco-benefício na utilização de antipsicóticos de segunda geração para o tratamento do factor psicose e agitação na demência. No entanto, vários ensaios mostram que, quer o haloperidol, quer os antipsicóticos de segunda geração, melhoram os sintomas de agitação-psicose na DA. ${ }^{12}$ Todavia, há vários estudos que mostram que estes fármacos estão associados a um aumento da mortalidade (especialmente por acidentes cérebro- e cardiovasculares, e infecções) ${ }^{13,14} \mathrm{e}$ uma eficácia baixa. Por estas razões, a agência americana Food and Drug Administration (FDA), e posteriormente a europeia European Medicines Agency (EMEA, actual EMA) realçaram que estes fármacos não estavam aprovados para o tratamento dos SPCD. Todavia, outras análises parecem demonstrar que esse risco não é muito diferente para o haloperidol e, mais importante, é tanto maior quanto pior for a condição dos doentes. Parece, assim, que estes fármacos são de evitar em doentes muito fragilizados, com comorbilidades somáticas importantes. A sua prescrição deve ser discutida com os familiares e obtida a sua autorização oral (que deve ser registada no processo). A continuação do tratamento deve ser acompanhada de perto e devem ser descontinuados logo que possível.

É relativamente consensual que os antipsicóticos devem ser evitados na demência com corpos de Lewy, pois estes doentes apresentam uma grande sensibilidade a estes fármacos. Todavia, parece que os antipsicóticos de segunda geração com menos propensão a provocar efeitos extra-piramidais, como sejam a clozapina e a quetiapina em doses baixas (25-75 mg/dia, embora possam ser inferiores, ou superiores), poderão apresentar algum benefício. É obrigatória a realização do controlo hematológico definido durante a terapêutica com clozapina, sendo um erro muito grave a prescrição de clozapina sem esta monitorização, pelo risco de morte associado a agranulocitose.

Um dos sintomas da depressão no idoso pode ser a agitação psicomotora, daí que os antidepressivos também podem ser eficazes. O tratamento da depressão na demência está discutido na secção respectiva.

Os anticonvulsivantes têm uma eficácia inconsis-tente, mas é nossa opinião que têm indicação pontual e devem ser usados para casos graves e por especialistas.

\section{Cluster depressão/ansiedade}

Existem poucos estudos referentes às terapêuticas não farmacológicas para a depressão. Recomenda-se que as pessoas possam tomar decisões pessoais, no que toca à escolha das roupas ou das refeições, sejam envolvidas em actividades que dêem prazer e, tanto quanto possível, personalizadas, e se evite o isolamento social (a melhor forma é interagir com pessoas significativas, se possível). 
Farmacologicamente, o tratamento da depressão na demência deve ser idêntico ao tratamento da depressão do idoso, mantendo-se, regra geral, as mesmas indicações. A depressão no idoso tem sido associada ao aumento da mortalidade por várias causas e, por isso, deve ser tratada na totalidade. Todavia, as doses são habitualmente menores, é maior o tempo para atingir os resultados terapêuticos e há maior sensibilidade a alguns efeitos adversos. Volta-se a referir a importância de não serem deixados sintomas residuais, já que estes estão associados à maior probabilidade de recaída e recorrência.

São claramente preferíveis os inibidores selectivos da recaptação da serotonina (SSRIs), pelos baixos riscos cardiovasculares, embora também existam riscos de quedas, hiponatrémia, perda de peso e interacções medicamentosas (nomeadamente com a varfarina). Se existirem importantes problemas de sono e falta de apetite, com baixo peso, e ausência de diabetes ou hipercolesterolémia não controlada, a mirtazapina é uma excelente escolha. Embora só deva ser feita por médicos com experiência, a sua combinação com SSRIs é habitualmente bem tolerada e eficaz para casos mais graves.

Para casos mais graves, ou de cariz melancólico, podem ser usados os inibidores da recaptação da noradrenalina e da serotonina, como a venlafaxina, embora este fármaco apresente menor segurança nos idosos.

Os SSRIs devem ser iniciados com uma dose baixa para evitar os efeitos adversos gastrointestinais, mas esta tem de ser aumentada ao fim de poucos dias para a dose mínima eficaz estabelecida e então aguardar 4 semanas para a resposta. Se esta não for satisfatória deve ser aumentada até à dose que provoque a remissão dos sintomas, obviamente ponderando os efeitos adversos. Não se devem manter doses baixas subterapêuticas, sendo preferível pedir aconselhamento se o tratamento não estiver a ser eficaz. Ao fim de seis meses após a remissão dos sintomas, deve ser iniciada a retirada do fármaco antidepressivo, tendo em atenção que pode surgir síndrome de abstinência, especialmente para os fármacos de semi-vida mais curta.

Para o tratamento da ansiedade, podem ser usadas benzodiazepinas, com as precauções atrás referidas, ou a buspirona. A buspirona, desde que em doses adequadas (pelo menos $30 \mathrm{mg}$ por dia) e após um período de tempo adequado (mais de 4 a 6 semanas) é uma ex- celente opção para a terapêutica da ansiedade crónica diurna, pois não tem potencial de dependência, carece de propriedades sedativas e é praticamente desprovida de efeitos adversos cardíacos. Não deve ser usada em insuficientes renais.

\section{Alterações do sono}

As medidas não farmacológicas indicadas são a higiene de sono (com hora rígida para levantar, evitar estimulantes como o café ou o chá, proibir em geral sestas durante o dia), manter actividade física diária, com exposição luminosa, e manter uma luz de presença acesa durante a noite.

Em termos farmacológicos, existem à disposição as benzodiazepinas mais sedativas de semi-vida curta (como o lorazepam 0,5-2 mg à noite se for iniciado de novo), o zolpidem (com menor possibilidade de quedas e confusão diurna) e a trazodona. A melatonina, apesar de parecer ser uma boa opção, não revelou eficácia na DA, nos ensaios mais bem desenhados, nem sobre a agitação, nem sobre o sono. Se houver agitação nocturna durante o sono, ou para as alterações do sono da demência com corpos de Lewy, o clonazepam também é uma opção.

Estes fármacos devem ser iniciados com uma dose mínima, que deve ser aumentada até se obter resposta.

\section{REFERENCIAÇÃO}

Qualquer SPCD de difícil controlo, especialmente se não melhorar após as medidas terapêuticas aqui descritas, poderá ser referenciado a uma consulta de Psiquiatria ou Neurologia, com a indicação de «SPCD [especificar] de difícil controlo após tratamento com [especificar]».

\section{REFERÊNCIAS BIBLIOGRÁFICAS}

1. Brodaty H, Finkel S. Behavioral and Psychological Symptoms of Dementia (BPSD), Educational Pack. Northfield, IL: International Psychogeriatric Association; 2002.

2. Simões do Couto $F$, Verdelho $A$, Mendonça A. Demência. In: Ponce $P$, coord. Manual de Terapêutica Médica. Lisboa: Lidel; 2010.

3. Lawlor B. Sintomas comportamentais e psicológicos das demências. In: Firmino H, editor. Psicogeriatria. Coimbra: Psiquiatria Clínica; 2006. p. 267-79.

4. National Institute for Health and Clínical Excelence Dementia. A NICE-SCIE guideline on supporting people with dementia and their carers in health and social care. London: The British Psychological Society; 2007. 
5. Waldemar G, Dubois B, Emre M, Georges J, McKeith IG, Rossor M, et al. Recommendations for the diagnosis and management of Alzheimer's disease and other disorders associated with dementia: EFNS guideline. Eur J Neurol 2007 Jan; 14 (1):e1-26.

6. Cummings LJ. The neuropsychiatry of dementing disorders. In: Cummings LJ, editor. The neuropsychiatry of Alzheimer s disease and related dementias. LA: Martin Dunitz; 2003. p. 2-14.

7. Cummings JL, Mega M, Gray K, Rosenberg-Thompson S, Carusi DA, Gornbein J. The neuropsyhiatric inventory: comprehensive assessment of psycopathology in dementia. Neurology 1994 Dec; 44 (12): 230814.

8. Grupo de Estudos de Envelhecimento Cerebral e Demências. Escalas e Testes na Demência. $2^{\text {a }}$ ed. Lisboa: Grupo de Estudos de Envelhecimento Cerebral e Demências; 2008.

9. Frisoni $G B$, Rozzini L, Gozzetti A, Binetti G, Zanetti O, Bianchetti A, et al. Behavioral syndromes in Alzheimer's disease: description and correlates. Dement Geriatr Cogn Disord 1999 Mar-Apr; 10 (2): 130-8.

10. Fu C, Chute DJ, Farag ES, Garakian J, Cummings JL, Vinters HV. Comorbidity in dementia: an autopsy study. Arch Pathol Lab Med 2004 Jan; 128 (1): 32-8.

11. Pereira AF, Simões do Couto F, de Mendonca A. The use of laboratory tests in patients with mild cognitive impairment. J Alzheimers Dis 2006 Sep; 10 (1): 53-8.
12. Sultzer DL, Gray KF, Gunay I, Berisford MA, Mahler ME. A double-blind comparasion of trazodone and haloperidol for treatment of agitation in patients with dementia. Am J Geriat Psychiatry 1997 Winter; 5 (1): 60-69.

13. Ballard C, Hanney ML, Theodoulou M, Douglas S, McShane R, Kossakowski K, et al. The dementia antipsychotic withdrawal trial (DART$A D)$ : long-term follow-up of a randomised placebo-controlled trial. Lancet Neurol 2009 Feb; 8 (2): 151-7.

14. Schneider LS, Tariot PN, Dagerman KS, Davis SM, Hsiao JK, Ismail MS, et al.; CATIE-AD Study Group. Effectiveness of atypical antipsychotic drugs in patients with Alzheimer's disease. N Engl J Med 2006 Oct 12; 355 (15): 1525-38.

Os autores declararam não possuir conflitos de interesses

\section{ENDEREÇO PARA CORRESPONDÊNCIA}

Instituto de Farmacologia e Neurociências

Instituto de Medicina Molecular

Faculdade de Medicina da Universidade de Lisboa

Av. Prof. Egas Moniz

1649-028 Lisboa

\section{ABSTRACT}

\section{BEHAVIORAL AND PSYCHIATRIC SYMPTOMS OF DEMENTIA}

Besides cognitive changes, dementia disorders have other very important symptoms. Some of these symptoms, that include an array of behavioral and psychiatric manifestations, are known as behavioral and psychiatric (or psychological) symptoms of dementia (BPSD). For several reasons they are an important case for General Practitioner consultation

Some of these symptoms tend to occur simultaneously, in factors, such as psychosis (agitation and aggressiveness), mood (depression and anxiety), frontal behavior (desinhibition and euphoria), apathy and insomnia.

The cause of these symptoms can be dementia itself, and in some situations they are a core diagnostic feature in the diagnosis. However, the possibility of a drug or a somatic disease as a cause for these symptoms must always be actively sought, especially if the BPSD have a particular clinical presentation. So, the exclusion of another medial disorder (that is non demential) is mandatory.

After excluding other causes, a treatment with an anti-dementia drug must be offered, as there is evidence that acetylcholinesterase inhibitors and memantine improve, not only cognition, but also BPSD in some dementia (including Alzheimer $s$ disease). Then, non-pharmacologial measures must be started. However, a pharmacological treatment with psychotropic drugs should also be started, if treatment is urgent or the symptoms severe.

The symptom or symptoms should be identified and the therapeutic targeted for those specific symptoms. Despite the clinical trials performed with psychotropics for BPSD are uncommon and sometimes have questionable designs, it is very important to treat these symptoms. Caregivers should also be instructed on how to deal with BPSD. Whenever a BPSD is difficult to control, especially if not improving after a trial with the measures here described, a referral for Neurologic or Psychiatric evaluation should be done. The referral note should be "BPSD [specify] severe and not improving after treatment with [specify]".

Keywords: Behavioral and Psychiatric Symptoms of Dementia; Diagnosis; Treatment; Antipsychotics; Psychotropics. 